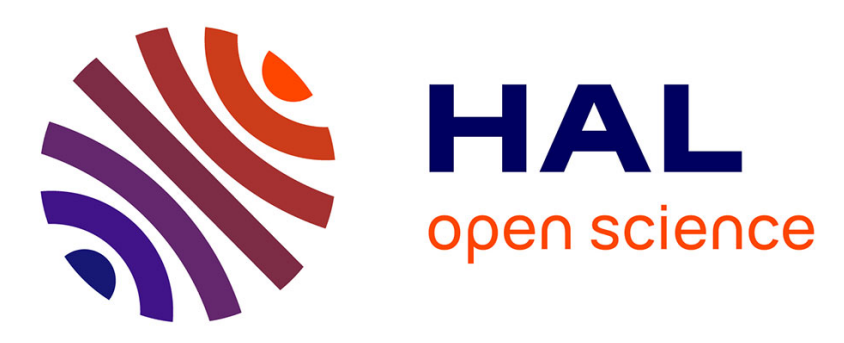

\title{
Feedback law with Probabilistic Certification for Propofol-Based Control of BIS During Anesthesia
}

Mazen Alamir, Mirko Fiacchini, Isabelle Queinnec, Sophie Tarbouriech, Michel Mazerolles

\section{- To cite this version:}

Mazen Alamir, Mirko Fiacchini, Isabelle Queinnec, Sophie Tarbouriech, Michel Mazerolles. Feedback law with Probabilistic Certification for Propofol-Based Control of BIS During Anesthesia. International Journal of Robust and Nonlinear Control, 2018, 28 (18), pp.6254-6266. 10.1002/rnc.4374 . hal-01335969

\section{HAL Id: hal-01335969 \\ https://hal.science/hal-01335969}

Submitted on 22 Jun 2016

HAL is a multi-disciplinary open access archive for the deposit and dissemination of scientific research documents, whether they are published or not. The documents may come from teaching and research institutions in France or abroad, or from public or private research centers.
L'archive ouverte pluridisciplinaire HAL, est destinée au dépôt et à la diffusion de documents scientifiques de niveau recherche, publiés ou non, émanant des établissements d'enseignement et de recherche français ou étrangers, des laboratoires publics ou privés. 


\title{
Feedback law with Probabilistic Certification for Propofol-Based Control of BIS During Anesthesia
}

\author{
Mazen Alamir* Mirko Fiacchini* Isabelle Queinnec ** \\ Sophie Tarbouriech ${ }^{* *}$ Michel Mazerolles ${ }^{* * *}$ \\ * Gipsa-lab, CNRS-Control Systems department, University of \\ Grenoble Alpes.Email: mazen.alamir(mirko.fiacchini)@grenoble-inp.fr. \\ ** LAAS-CNRS, Université de Toulouse, CNRS, Toulouse, France. \\ Email isabelle.queinnec(sophie.tarbouriech)@laas.fr. \\ *** Département d'anesthésie-réanimation, CHU Toulouse, 31059 \\ cedex, France.Email mazerolles.m@chu-toulouse.fr
}

\begin{abstract}
This paper proposes a new control strategy for Propofol injection during anesthesia in patients undergoing surgery and where the bispectral index (BIS) is considered to be the regulated variable. The proposed control shows the nice feature of being completely independent of the knowledge of the pharmacokinetics that govern the diffusion of the drug. The paper also proposes a certification framework that gives a probabilistic guarantee regarding the containment of the BIS inside the desired interval as well as for the time needed for the BIS to be steered to this interval. Moreover, this certification is given for realistic (and hence very high) level of uncertainties on the parameters that define the unknown-to-the-controller dynamics. This last feature is checked using a widely used model.
\end{abstract}

Keywords: Anesthesia, BIS, Propofol, Robust Feedback, Performance Certification.

\section{INTRODUCTION}

When it comes to use feedback strategies to monitor drug delivery on humans, the concept of robustness cannot be overestimated. This is because, unlike human designed technological systems that can be tightly modeled using well established principles, modeling the dynamics of the underlying biological processes is very difficult. Even when potentially faithful models are derived (which is rather rare), the parameters that such models involve are highly uncertain. Therefore, any candidate model-based feedback strategy that is intended to rationalize the drug delivery has to assess the robustness of its performance against these unavoidable and particularly high uncertainties.

The automatic control of anesthesia makes no exception. Many advances have been achieved since the early expert systems (O'Hara et al., 1992). The benefit from a successful automated process is twofold: 1) It avoids the dramatic consequence of a possible transient lack of attention of the anesthetist. 2) It reduces the sensitivity to initial estimation of the specific patient responsiveness to the drug (or even ideally suppresses the need for it). This second feature needs the feedback strategy to not heavily rely on the knowledge of the specific patient parameters.

In this paper, focus is concentrated on the control of deep anesthesia via Propofol injection. More precisely, as there is no direct measurement of the depth of anesthesia, the Bispectral Index (BIS) which is a commercially available EEG-based index, is used as a surrogate measure for the depth of anesthesia. Notice however that the BIS is one among multiple indicators that the anesthetist keeps monitoring during the operation (Charbati et al., 2009; Dumont, 2012).

Since the early works by (Bickford, 1951; Ross et al., 1982; Schwilden et al., 1987; Ritchie et al., 1987; Mortier et al., 1998) that assessed the benefit from closed-loop feedback mode when compared to standard practices, several feedback design strategies have been proposed for the above problem (Ionescu et al., 2008; Zabi et al., 2015; Smet et al., 2007). More precisely, a predictive control strategy based on a nominal linear model is proposed in (Ionescu et al., 2008). The robustness of the resulting closed-loop behavior is tested by simulating a dozen of different sets of parameters used in the model. The performance and the robustness of this law showed comparable to the adaptive controller proposed in (Vishnoi and Roy, 1991; Smet et al., 2007; Gentilini et al., 2001) which is based on on-line adaptation of the underlying model through a preliminary (open-loop) induction phase. More recently, a dynamic decoupling approach is used in (Zabi et al., 2015) where a Linear Matrix Inequality (LMI) that handles polytopic uncertainties is used to design a robust feedback law. The robustness is assessed through several simulations with various parameter sets.

Most of the above cited works are based on the use of linear model structure. This assumption simplifies the controller design but also enables rigorous robustness certification (as far as the linearity assumption is relevant) via Linear 
Matrix Inequalities (LMI's) or set-invariance frameworks. In most of these works, focus is made on the proof of stability although this is intrinsically an asymptotic property. For finite time processes such as the one we are interested in where finite time surgery is involved, the asymptotic stability is not the appropriate paradigm. Instead, one should focus on the time needed to reach the desired region for BIS and whether or not the latter remains inside this region for the remaining time. Moreover, the robustness in the above cited works is taken in the traditional worst-case sense which is generally too stringent as even potentially extremely unlikely configuration of parameters are taken into account.

In this work, a different approach is adopted in which the linearity assumption is dropped and certification is obtained by means of randomized optimization. More precisely, the recently developed control scheme (Alamir, 2015), originally developed for saturated control of unknown scalar systems, is used in a somehow back-stepping form. The parameters of the resulting control scheme are then optimized via the randomized optimization framework (Alamo et al., 2009) in order to get certified closedloop performance in terms of:

- The maximal time needed before entering the final $(40-60)$ region for the BIS indicator.

- The effective invariance of this terminal BIS region.

- The fact that BIS is kept above 40 all the time.

The certification mentioned above needs a simulation model (that is unknown to the controller) in order to perform the randomized optimization. To this end, some existing models are used to derive highly uncertain simulators in order to support the certification claims. The results suggest extremely high robustness to the parameters of the pharmacokinetics model (up to $400 \%$ of uncertainties on all parameters).

The paper is organized as follows: Section 2 states the problem addressed in the paper. A brief sketch of the solution is derived in Section 3. Since the proposed solution is based on two ingredients, which are the control framework of (Alamir, 2015) and the randomized certification framework of (Alamo et al., 2009), these two results are recalled in Section 4. The proposed feedback strategy is proposed in Section 5 while Section 6 gives extensive closed-loop simulations and the certification results. Finally, Section 7 concludes the paper and gives hints for further investigations.

\section{PROBLEM STATEMENT}

In this paper, we consider the problem of steering and maintaining the BIS level to and inside the interval

$$
\mathcal{I}:=\left[50-\delta_{B}, 50+\delta_{B}\right]
$$

by means of appropriate feedback-based Propofol injection strategy. It is assumed that a nonlinear static map, denoted hereafter by $\Psi_{B}$ links the BIS to the drug concentration $C_{e}$ in the effect site compartment, namely:

$$
\mathrm{BIS}=\Psi_{B}\left(C_{e} \mid p_{B}\right)
$$

where $p_{B}$ are uncertain modeling parameters. The map $\Psi_{B}$ is supposed to be invertible so that one can write:

$$
C_{e}=\Psi_{B}^{-1}\left(\mathrm{BIS} \mid p_{B}\right)
$$

The dynamics of $C_{e}$ is governed by (Bailey and Haddad, 2005):

$$
\dot{C}_{e}(t)=k_{e 0}\left[C_{p}(t)-C_{e}(t)\right]
$$

in which $C_{p}$ represents the concentration of drug in the central compartment (blood). This is a common modeling compartmental approach in which only the drug quantity inside the effect site compartment is effective while equation (4) models the transfer between the blood and the site effect compartment. This models the diffusion-induced dynamics between the injection of the drug and its effect on the BIS indicator.

Finally, the dynamics of $C_{p}$ is given by:

$$
\dot{C}_{p}(t)=\Phi\left(\xi(t), C_{e}(t), C_{p}(t)\right)+v(t)
$$

where $\xi \in \mathbb{R}^{n_{\xi}}$ is a varying non measured quantity with unknown dynamics while $\Phi$ is an unknown function. This term gathers the leaks of drug from the blood to the well and the poorly perfused body tissues. $v$ stands for the drug injection intensity which belongs to the admissible set $\mathbb{V}:=[0, \bar{v}]$ where $\bar{v}$ is the maximum injection intensity.

This paper is about designing a feedback law that does not use any knowledge regarding the term $\Phi(\cdot)$ involved in (5). Nevertheless, in order to support simulations AND certification tasks, we need relevant realization of this term. Concrete choices for such realizations are described in Section 6. At this stage of the presentation and in order to keep the problem statement at a generic level, the following Definition is needed:

Definition 1. (Relevant Model Completion (RMC)). Consider the following items:

(1) A dynamic equation of the form:

$$
\dot{\xi}=F\left(\xi, C_{e}, C_{p}, v, p_{\phi}\right) \quad ; \quad \xi \in \mathbb{R}^{n_{\xi}}
$$

where $p_{\phi} \in \mathbb{P} \subset \mathbb{R}^{n_{\phi}}$ is a vector of parameters that belongs to an admissible set $\mathbb{P}$.

(2) A static map $\Phi: \mathbb{R}^{n_{\xi}} \times \mathbb{R}^{2} \rightarrow \mathbb{R}$;

(3) A probability distribution $\mathcal{P}_{\phi}$ that enables to get random realizations of the parameter vector $p_{\phi}$ inside the admissible set $\mathbb{P}$;

(4) Two probability distributions $\mathcal{P}_{B}$ and $\mathcal{P}_{e 0}$ that enables to get random realizations of $p_{B}$ and $k_{e 0}$ involved in equations (2) and (4) respectively.

We say that the preceding items define a Relevant Model Completion if the family of dynamic models defined by (2), (4), (5) completed by (6) is a relevant model to describe the Propofol-based action on the BIS indicator including the inter-patient variability as far as $p_{B}, k_{e 0}$ and $p_{\phi}$ are fired according to the probability distributions $\mathcal{P}_{B}, \mathcal{P}_{e 0}$ and $\mathcal{P}_{\phi}$ respectively.

Based on the description above, the control problem addressed in the present paper can be described as follows:

Problem statement: Assuming that:

$\checkmark$ The BIS is measured;

$\checkmark$ A Relevant Model Completion is given;

$\checkmark$ A duration $T$ for the surgery is given.

The objective is twofold: 
(1) Define a dynamic control feedback of the form:

$$
\begin{aligned}
& \dot{z}=g(z, \mathrm{BIS}) \\
& v=K(z, \mathrm{BIS})
\end{aligned}
$$

that is designed to steer the BIS inside the interval $\mathcal{I}$ after a finite time $\tau_{r}$ and maintain it inside $\mathcal{I}$ over the remaining time $\left[\tau_{r}, T\right]$ while never cross the lower bound $0.5-\delta_{B}=40$ on the BIS level.

(2) Provide an upper bound $\bar{\tau}_{r}>0$ such that the following condition holds on the probability of $\tau_{r}$ being lower than $\bar{\tau}_{r}$ :

$$
\operatorname{Pr}\left[\tau_{r} \leq \bar{\tau}_{r}\right] \geq 1-\eta
$$

with acceptably small $\tau_{r}$ and a very small $\eta$.

In the following section, the general guidelines of the proposed methodology to address this problem are first sketched to get the whole picture before a detailed derivation is given in later sections.

\section{SKETCH OF THE PROPOSED SOLUTION}

The proposed methodology can be summarized in two steps:

(1) Design a feedback control law;

(2) Certify the properties of the corresponding closedloop behavior.

The following two sections explain these two steps.

\subsection{Feedback Design}

Let us denote $y=$ BIS the only measured quantity. Denote by $x:=\left(C_{e}, C_{p}\right)^{T}$ the state vector of the following dynamic model that is used in the control design and which is derived from (4)-(5):

$$
\begin{array}{lr}
\dot{x}_{1}=k_{e 0}\left(-x_{1}+x_{2}\right) & k_{e 0} \text { uncertain } \\
\dot{x}_{2}=\Phi+v & \Phi \text { unknown }
\end{array}
$$

Let us also denote by $p$ the vector that concatenates all the uncertain parameters while $p_{c}$ gathers the uncertain parameters that are not associated to $\Phi$, namely

$$
p:=\left[\begin{array}{l}
p_{c} \\
p_{\phi}
\end{array}\right] \quad ; \quad p_{c}:=\left[\begin{array}{l}
p_{B} \\
k_{e 0}
\end{array}\right]
$$

Finally, the notation $\mathcal{P}$ designates the probability distribution of $p$ that is induced by $\mathcal{P}_{B}, \mathcal{P}_{e 0}$ and $\mathcal{P}_{\phi}$.

Assume a nominal parameter vector $p_{c}^{\text {nom }}:=\left(p_{B}^{\text {nom }}, k_{e 0}^{\text {nom }}\right)$ of $p_{c}$. Using $p_{B}^{\text {nom }}$, equation (3) can be used as a measurement equation for $x_{1}=C_{e}=\Psi^{-1}\left(y \mid p_{B}^{n o m}\right)$. This measurement together with (9a) can be used to estimate $x_{2}$. More precisely, a Luenberger observer can be designed to produce an estimation $\hat{x}_{2}$ of $x_{2}$, namely:

$$
\dot{\hat{x}}=\left[\begin{array}{cc}
-k_{e 0}^{n o m} & k_{e 0}^{n o m} \\
0 & 0
\end{array}\right] \hat{x}+\left[\begin{array}{l}
0 \\
v
\end{array}\right]+\left[\begin{array}{l}
L_{1} \\
L_{2}
\end{array}\right]\left[\Psi_{B}^{-1}\left(y \mid p_{B}^{n o m}\right)-\hat{x}_{1}\right]
$$

where the matrix $L=\left[\begin{array}{ll}L_{1} & L_{2}\end{array}\right]^{T}$ is a standard Luenberger observer gain (Antsaklis and Michel, 2007). Note that in this observer, the assumption $\Phi=0$ is used, which can still lead to acceptable observer performance provided that sufficiently high gain $L$ is used. This is a standard practice in extended-state-based observer.
Now that $x_{1}$ is measured and $x_{2}$ can be reconstructed, one can focus on the design of a state feedback for (9a)(9b) knowing that once such a feedback is designed, the estimated state can be used. The objective of the control design will be to steer the state $x$ to the desired steady state that is defined by:

$$
x^{d}:=\left[\begin{array}{l}
1 \\
1
\end{array}\right] \Psi^{-1}\left(50 \mid p_{B}^{\text {nom }}\right)
$$

which is simply the steady state of (9a)-(9b) that is compatible with the desired BIS value, namely 50 .

The design of the control law uses a technique that is inspired by the well known back-stepping approach. In order to better understand the idea, let us rewrite (9a)(9b) in the following form:

$$
\begin{aligned}
& \dot{x}_{1}=k_{e 0}\left[-x_{1}-e_{2}+x_{2}^{r e f}\right] \\
& \dot{x}_{2}=\Phi+v
\end{aligned}
$$

where $e_{2}=x_{2}^{r e f}-x_{2}$ in which $x_{2}^{r e f}$ is some reference value for $x_{2}$ to be designed in the sequel. In the back-stepping approach, one views $x_{2}^{r e f}$ as a control signal that regulates $x_{1}$ at $x_{1}^{d}$ and views $v$ as a control signal that regulates $x_{2}$ at $x_{2}^{r e f}$. By doing so, the control problem can be split into two scalar control problems in each of which the dynamic scalar controlled system takes the following form:

$$
\dot{x}_{i}=\alpha_{i}\left[u_{i}-h_{i}\right]
$$

where $\alpha_{i}$ and $h_{i}$ are given by:

$$
\left(\alpha_{1}, \alpha_{2}\right)=\left(k_{e 0}, 1\right) \quad ; \quad\left(h_{1}, h_{2}\right)=\left(x_{1}+e_{2},-\Phi\right)
$$

Therefore, one can apply the above back-stepping inspired idea provided that a feedback design can be systematically obtained for systems of the form (14) in which $\alpha_{i}$ and $h_{i}$ are partially unknown. This is because $\Phi, e_{2}$ and $k_{e 0}$ are supposed to be (partially or totally) unknown for their respective controllers.

In the next section, a recent result (Alamir, 2015) is recalled regarding a systematic control design for uncertain systems of the form (14). This result is then used to give an explicit feedback design. For the time being and in order to keep the general overview of the proposed solution, let us assume that some dynamic output feedback design is achieved so that a dynamic control of the form (7) is obtained leading to the following closed-loop system (recall that $y=\mathrm{BIS})$ :

$$
\begin{aligned}
\dot{x}_{1} & =k_{e 0}\left(-x_{1}+x_{2}\right) \\
\dot{x}_{2} & =\Phi+K(z, y) \\
\dot{z} & =g(z, y)
\end{aligned}
$$

Notice that the definition of this system is incomplete as the dynamics of $\Phi$ is unknown. However the definition of the feedback $K(z, y)$ does not involve any knowledge regarding this dynamics. Now as soon as a RMC is defined in the sense of Definition 1, the equations (16a)-(16c) can be completed to yield an autonomous closed-loop system for each choice of the parameter vector $p$, namely:

$$
\begin{aligned}
\dot{x}_{1} & =k_{e 0}\left(-x_{1}+x_{2}\right) \\
\dot{x}_{2} & =\Phi\left(\xi, x_{1}, x_{2}\right)+K(z, y) \\
\dot{z} & =g(z, y) \\
\dot{\xi} & =F\left(\xi, x_{1}, x_{2}, K(z, y), p_{\phi}\right) \\
y & =\operatorname{BIS}=\Psi_{B}\left(x_{1} \mid p_{B}\right)
\end{aligned}
$$


which is a complete simulator for the specific closed-loop patient that is defined by the value of $p:=\left(p_{B}^{T}, k_{e 0}, p_{\phi}\right)^{T}$.

Remark 1. Note that nominal values $k_{e 0}^{\text {nom }}$ and $p_{B}^{\text {nom }}$ used in the design of the observer [see (11)] and the definition of the desired state $x^{d}$ [see (12)] are implicitly contained in the functions $K(z, y)$ and $g(z, y)$.

The complete definition of the feedback law is given in Section 5 .

\subsection{Probabilistic certification}

The simulator defined by (17a)-(17e) gives the evolution of the BIS indicator during the surgery when the feedback law is applied and for a specific value $p$ of the vector of parameters that is related to a specific patient. Let us denote by $y(t \mid p), t \in[0, T]$ the resulting trajectory for the BIS indicator. Therefore, it is possible to define the response time $\tau_{r}(p)$ for that specific value of $p$ by:

$\tau_{r}(p):= \begin{cases}t^{*}:=\min \{\bar{t} \mid y(t \mid p) \in \mathcal{I} \forall t \in[\bar{t}, T]\} & \text { if } t^{*} \text { exists } \\ 2 T & \text { otherwise }\end{cases}$

that is to say, if there is $\bar{t}$ such that for all $t \in[\bar{t}, T]$, the BIS remains inside the targeted region $\mathcal{I}$ [see (1)], then $\tau_{r}(p)$ is set to the least of such values, otherwise, it is set to $2 T$. Note that the use of $2 T$ in the second branch of (18) is arbitrary and simply means that the admissible interval $\mathcal{I}$ is not reached during the surgery.

The probabilistic certification paradigm is linked to the possibility to have something to say about the issue of the operation on the whole population of patients, represented by the possible values of $p$ associated to the probability distribution $\mathcal{P}$ defined by the RMC under interest. More precisely, one seeks a way to be able to certify that the following probability holds:

$$
\operatorname{Pr}_{\mathcal{P}}\left[\tau_{r}(p) \leq \bar{\tau}_{r}\right] \geq 1-\eta
$$

with sufficiently small upper bound $\bar{\tau}_{r}$ and sufficiently small probability of failure $\eta$. The way such result can be obtained is based on the probabilistic certification framework that is recalled in Section 4.

\section{RECALLS ON SOME RELEVANT RESULTS}

This section contains some recalls on two results that are used to complete the proposed solution. The first one concerns the control design for scalar uncertain systems of the form (14). The second one concerns the concept of probabilistic certification. These are the subject of the following two sub-sections.

\subsection{Constrained Feedback of Uncertain Scalar Systems}

Let us consider a general system of the form (14):

$$
\dot{x}=\alpha[u-h]
$$

in which $\alpha$ and $h$ are unknown, $u \in\left[u_{\min }, u_{\max }\right]$. It is assumed that $\alpha \geq \alpha_{\min }>0$ while $h \in\left[h_{\min }, h_{\max }\right]$ where the bounds are assumed to be known and such that the following inequalities hold:

$$
\begin{gathered}
u_{\max }-h_{\max } \geq \varrho_{+}>0 \\
h_{\min }-u_{\min } \geq \varrho_{-}>0
\end{gathered}
$$

with some positive scalars $\varrho_{+}$and $\varrho_{-}$. It simply means that the control has sufficient authority. Consider the following saturation function on the control variable:

$$
S(u):= \begin{cases}u_{\max } & \text { if } u \geq u_{\max } \\ u_{\min } & \text { if } u \leq u_{\min } \\ u & \text { otherwise }\end{cases}
$$

Using the above notation and assumptions, the following result can be proved (Alamir, 2015):

Proposition 2. Take some $\lambda>0$. Take any $\lambda_{f}>0$ satisfying:

$$
\lambda_{f}<\left[\min \left\{\frac{\min \left\{\varrho_{+}, \varrho_{-}\right\}}{u_{\max }-u_{\min }}, \frac{\alpha_{\min }}{4}\right\}\right] \times \lambda
$$

If the following conditions hold:

(1) $x_{d}$ is constant and

(2) the dynamics of the unknown term $h$ satisfies:

$$
\left|\frac{d h}{d t}\right| \leq \delta_{h}
$$

then the dynamic state feedback law defined by:

$$
\begin{aligned}
& \dot{z}=\lambda_{f}\left[S\left(\lambda\left(x_{d}-x\right)+z\right)-z\right] \\
& u=S\left(\lambda\left(x_{d}-x\right)+z\right)
\end{aligned}
$$

leads to a tracking error $e_{x}=x-x_{d}$ that satisfies:

$$
\lim _{t \rightarrow \infty}\left|x(t)-x_{d}\right| \leq \frac{\delta_{h}}{\lambda \lambda_{f}}
$$

Note that this result means that for constant unknown $h$, asymptotic convergence results. Moreover, even with dynamic unknown $h$, the value of $\lambda$ can always be taken sufficiently high to enforce any asymptotic small error. In Section 5 this result is used to derive a dynamic backstepping like state feedback for the system (13a)-(13b).

\subsection{Probabilistic Certification}

Recall that according to the discussion of Section 3.2, one looks for a solution (if any) to the following optimization problem:

$$
\min _{\bar{\tau}_{r}} J\left(\bar{\tau}_{r}\right):=\bar{\tau}_{r} \quad \mid \quad \operatorname{Pr}\left[\begin{array}{c}
\tau_{r}(p) \leq \bar{\tau}_{r} \\
\mathrm{AND} \\
\min _{t \in[0, T]} y(t \mid p) \geq 40
\end{array}\right] \geq 1-\eta
$$

This is a rather intractable formulation as the probability depends on a rather high dimensional vector $p$ of random parameters. The idea of probabilistic certification is to replace this problem by the following more tractable one:

$$
\min _{\bar{\tau}_{r}} J\left(\bar{\tau}_{r}\right):=\bar{\tau}_{r} \quad \mid \quad \sum_{i=1}^{N} I\left(\bar{\tau}_{r}, p^{(i)}\right) \leq m
$$

where

- $p^{(i)}, i=1, \ldots, N$ are $N$ random samples of $p$ which are obtained according to the distribution $\mathcal{P}$.

- $I\left(\bar{\tau}_{r}, p^{(i)}\right)$ is the constraint violation indicator:

$$
I\left(\bar{\tau}_{r}, p^{(i)}\right):=\left\{\begin{array}{l}
1 \text { if } \tau_{r}(p)>\bar{\tau}_{r} \text { or } \min _{t} y(t \mid p)<40 \\
0 \text { otherwise }
\end{array}\right.
$$




\begin{tabular}{lcccc}
\hline$n_{r}$ & $\eta=0.1$ & $\eta=0.05$ & $\eta=0.01$ & $\eta=0.001$ \\
\hline 1 & 132 & 264 & 1317 & 13164 \\
5 & 154 & 308 & 1536 & 15354 \\
10 & 163 & 326 & 1628 & 16280 \\
100 & 193 & 386 & 1930 & 19299 \\
1000 & 223 & 445 & 2225 & 22249 \\
10000 & 252 & 503 & 2515 & 25148 \\
\hline
\end{tabular}

Table 1. Evolution of the sample size $N$ (number of samples needed to achieve the certification) as a function of the precision $\eta$ and the cardinality $n_{r}$ of the design parameter set $\mathbb{T}$ (confidence parameter $\delta=10^{-3}$ and $m=1$ are used)

- $m$ is some integer such that

$$
\frac{m}{N} \leq \eta
$$

Roughly speaking, the condition (28) states that only $m$ samples inside the population of $N$ samples violate the constraint $\left(\tau_{r}(p) \leq \bar{\tau}_{r}\right.$ and $\left.\min _{t} y(t \mid p) \geq 40\right)$. Now, intuitively, one would expect that if a value $\bar{\tau}_{r}$ is found such that for a sufficiently high number of samples $N$, only a small number $m$ satisfying (30) violates the constraint then one can say that the probability that the constraint is violated is close to $\eta$.

The problem lies obviously in the terms (sufficiently high $N$ ) and (close to $\eta$ ). That is where the rigorous formalism of probabilistic certification (Alamo et al., 2009) comes into action. Indeed, it gives for a priori chosen $m$, a priori desired $\eta$ and a priori defined confidence parameter $\delta$ the number of samples $N$ that one has to use. Note that the confidence parameter $\delta$ is the probability with which (28) can hold while $(27)$ is violated. In other words, $1-\delta$ is the probability that one satisfies the intractable inequality (27) while checking only the tractable inequality (28).

Many results are given in (Alamo et al., 2009) under different conditions, here only a specific case of interest is considered in which the set of candidate values of $\bar{\tau}_{r}$ are supposed to belong to a discrete set of cardinality $n_{r}$, namely:

$$
\bar{\tau}_{r} \in \mathbb{T}:=\left\{\bar{\tau}_{1}, \ldots, \bar{\tau}_{n_{r}}\right\}
$$

In this case, the number of samples $N$ is given by the following formulae (Alamo et al., 2009):

$$
N \geq \frac{1}{\eta}\left(m+\ln \left(\frac{n_{r}}{\delta}\right)+\left(2 m \ln \left(\frac{n_{r}}{\delta}\right)\right)^{1 / 2}\right)
$$

The corresponding values of $N$ for $m=1$ and the confidence parameter $\delta=10^{-3}$ are given in Table 1 .

\section{THE PROPOSED FEEDBACK STRATEGY}

As explained in Section 3.1, the dynamic feedback law (25) is applied to the following two scalar uncertain systems in a back-stepping form:

(1) System (13a) in which the control is $x_{2}^{r e f}$, the state is $x_{1}$ and the reference is $x_{1}^{d}$ given by (12). The virtual control $x_{2}^{r e f}$ belongs to $\left[\underline{\gamma} x_{2}^{d}, \bar{\gamma} x_{2}^{d}\right]$ where $\underline{\gamma} \in(0,1)$ and $\bar{\gamma} \in[1,2]$. This obviously defines an interval of variation around the steady value $x_{2}^{d}$.
(2) System (13b) in which the control is $v$, the state is $x_{2}$ and the reference is $x_{2}^{r e f}$. The control $v$ belongs to $[0, \bar{v}]$.

Using (25a)-(25b) the controller is given by:

$$
\begin{aligned}
\dot{z}_{1} & =\lambda_{f_{1}}\left[S_{1}\left(\lambda_{1}\left(x_{1}^{d}-x_{1}\right)+z_{1}\right)-z_{1}\right] \\
\dot{z}_{2} & =\lambda_{f_{2}}\left[S_{2}\left(\lambda_{2}\left(x_{2}^{r e f}\left(x_{1}, z_{1}\right)-x_{2}\right)+z_{2}\right)-z_{2}\right] \\
v & =S_{2}\left(\lambda_{2}\left(x_{2}^{r e f}\left(x_{1}, z_{1}\right)-x_{2}\right)+z_{2}\right)
\end{aligned}
$$

where

$$
\begin{aligned}
x_{2}^{r e f}\left(x_{1}, z_{1}\right) & :=S_{1}\left(\lambda_{1}\left(x_{1}^{d}-x_{1}\right)+z_{1}\right) \\
S_{1}(r) & := \begin{cases}\gamma x_{2}^{d} \text { if } r<\bar{\gamma} x_{2}^{d} \\
\overline{\bar{\gamma}} x_{2}^{d} \text { if } r>\bar{\gamma} x_{2}^{d} \\
r \quad \text { otherwise }\end{cases} \\
S_{2}(r) & := \begin{cases}0 & \text { if } r<0 \\
\bar{v} & \text { if } r>\bar{v} \\
r & \text { otherwise }\end{cases}
\end{aligned}
$$

Notice that the feedback defined by (33) depends on the following vector of parameters:

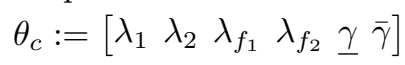

The feedback law given by $(33 \mathrm{c})$ can be put in the form $v=K(z, y)$ invoked in (16) and (17) once that $\left(x_{1}, x_{2}\right)$ is replaced by $\left(z_{3}, z_{4}\right):=\left(\hat{x}_{1}, \hat{x}_{2}\right)$ whose dynamics is given by the observer equation (11).

The last modification that is needed to take into account the uncertainties on the parameter $p_{B}$ consists in introducing an integral action. This is done by modifying the definition of the desired state $x^{d}$ from the original form (12) to the new form:

$$
x_{1}^{d}:=\Psi^{-1}\left(50+e \mid p_{B}^{n o m}\right)
$$

where the dynamics of the error $e$ is defined by:

$$
\dot{e}= \begin{cases}\lambda_{I}(50-y) & \text { if } y \in \mathcal{I} \\ 0 & \text { otherwise }\end{cases}
$$

where $\lambda_{I}>0$ is the integrator constant. This conditional definition enables the integrator state to be updated only in the vicinity of the desired BIS.

This completes the definition of the feedback scheme. In the next section, a specific widely used RMC is defined and the behavior of the resulting closed-loop system can be examined. In particular, certification results are obtained using the framework of Section 4.2.

\section{RESULTS}

This section is organized as follows:

(1) a specific RMC is first defined to serve in the simulations and the certification. Note that this definition includes the definition of the unknown map $\Phi$ and $F$ respectively given by (5) and (6), but also the definition of the admissible sets of the parameters $p_{c}$ and $p_{\phi}$ and their probability distributions. Any other model can be used in the framework without any change in the feedback design. Only the behavior and the quantitative results of the assessment would be different. 
(2) The set of control parameters defined by (34) and used in the feedback expression (33) is given. Note that these parameters can be optimized using the full randomized optimization framework of (Alamo et al., 2009). This would complicate the exposition of the methodology. Instead, we decided to assess the certification of the resulting closed loop for a specific hand-tuned control parameter. The very nice result suggests that optimization is not necessary here.

(3) Some temporal simulations for different parameter values $p$ are shown in order to show the widespread set of possible closed-loop trajectories.

(4) Finally, the upper bound $\bar{\tau}_{r}$ discussed in Section 4.2 is computed and the certification result is summarized.

These items are developed in the following sections.

\subsection{Definition of the RMC used for certification}

As an RMC, we shall consider the widely used compartmental model describing the dynamics of the drug in the human body [see (Ionescu et al., 2008; Zabi et al., 2015) and the references therein]. In this model, the twostates following dynamics is used to represent the unknown functions $\Phi$ and $F$ :

$$
\begin{aligned}
\dot{\xi}_{1} & =k_{12} x_{2}-k_{21} \xi_{1} \\
\dot{\xi}_{2} & =k_{13} x_{2}-k_{31} \xi_{2} \\
\Phi & =-\left(k_{10}+k_{12}+k_{13}\right) x_{2}+k_{21} \xi_{1}+k_{31} \xi_{2}
\end{aligned}
$$

where the coefficients $k_{i j}$ are given by nominal expressions that depend on the gender $(G)$, age $(A)$, height $(H)$ and weight $(W)$ of the patient:

$$
k_{i j}^{n o m}:=F_{i j}(G, A, H, W)
$$

Interested readers can consult (Ionescu et al., 2008) for the explicit expression of the functions $F_{i j}$. Recall that in the previous discussion, the vector gathering these parameters is the one denoted by $p_{\phi}$, namely:

$$
p_{\phi}:=\left[\begin{array}{lllll}
k_{10} & k_{12} & k_{21} & k_{13} & k_{31}
\end{array}\right] \in \mathbb{R}^{5}
$$

Note that equations (37a)-(37b) define the function $F$ invoked in (6). In this work, the probability distribution $\left(\mathcal{P}_{\phi}\right)$ associated to this parameter is defined by the following procedure to generate random samples:

(1) First the gender parameter $G$ is randomly chosen by equally probable Male/Female issue.

(2) Then, the continuous parameters $A \in[20,80], H \in$ $[140,200], W \in[50,110]$ are uniformly sampled inside the above defined intervals of age, weight and height. Then the corresponding parameter vector $p_{\phi}^{n o m}$ is computed by (38).

(3) The results of the two previous steps are then randomly perturbed by multiplying each parameter $p_{\phi_{i}}$, $i=1, \ldots, 5$ independently by a factor $\beta_{i}$ that is uniformly randomly chosen in the interval $[\beta, \bar{\beta}]$ where $\beta \in(0,1)$ and $\bar{\beta}>1$ :

$$
p_{\phi_{i}}=\beta_{i} \times p_{\phi_{i}}^{\text {nom }}(G, A, H, W) \quad \beta_{i} \in[\underline{\beta}, \bar{\beta}]
$$

The values $\beta=0.2$ and $\bar{\beta}=4$ are used in the following investigation. Note that this means that
Each parameter can randomly take any value between $20 \%$ and $400 \%$ of its nominal value. These nominal values are themselves randomly obtained through random samples of $G, A, H$, and $W$.

The static map between the BIS and $x_{1}$ is classically defined by (Ionescu et al., 2008):

$$
\mathrm{BIS}=\Psi_{B}\left(x_{1} \mid p_{B}\right):=100\left[1-\frac{x_{1}^{\gamma}}{x_{1}^{\gamma}+E_{c 50}^{\gamma}}\right]
$$

which is a model that is defined by the parameter vector $p_{B}:=\left(\gamma, E_{c 50}\right)$ for which, the following nominal value is used:

$$
p_{B}^{\text {nom }}:=(2.39,5.6)
$$

while the random sampling (distribution $\mathcal{P}_{B}$ ) is defined by a uniform sampling in the interval defined by $\pm 15 \%$ around the nominal values of the components. Similarly the nominal value $k_{e 0}^{n o m}=0.474$ is used for $k_{e 0}$ involved in (9a) while the real value used in the simulation is uniformly randomly chosen in the $\pm 30 \%$ interval around the nominal value. This defines the sampling probability $\mathcal{P}_{e 0}$ invoked in Definition 1.

\subsection{Definition of the control parameters}

The following control parameters are used in the definition of the dynamic feedback (33)

$$
\begin{aligned}
& \lambda_{1}=40 ; \lambda_{2}=10 ; \lambda_{f_{1}}=0.01 \lambda_{1} ; \lambda_{f_{2}}=0.1 \lambda_{2} \\
& \underline{\gamma}=0.7 ; \bar{\gamma}=1.3 ; \bar{v}=200
\end{aligned}
$$

The integrator constant is taken equal to $\lambda_{I}=0.1$ in (36). The sampling period for the control is taken equal to $\tau=6$ sec.

It is important here to strengthen the fact that whether these parameters meet the theoretical requirements of Proposition 2 does not really matter. The last word is given by the certification phase, which evaluates the probability of success of the designed feedback regardless of whether it lies inside the theoretical, generally over-stringent, sufficient conditions of Proposition 2. The latter can be viewed as a source of inspiration of the control design that is candidate for certification statement.

\subsection{Time simulations for different patients}

The aim of this section is to show how different closed-loop feedback Propofol injection time profiles might result when patients with highly different parameters are used in the simulation. This does not correspond to any certification but enables to get a feeling regarding the efficiency of the proposed feedback in handling the lack of knowledge of the patient's response to Propofol administration. To show this, three patients are considered with the following relative discrepancies w.r.t. the nominal values of the parameters (the expressions have to be interpreted componentwise):

$$
\frac{p_{\phi}-p_{\phi}^{\text {nom }}}{\left|p_{\phi}^{\text {nom }}\right|} \in\left\{\left[\begin{array}{c}
162 \% \\
273 \% \\
23 \% \\
253 \% \\
211 \%
\end{array}\right],\left[\begin{array}{c}
180 \% \\
-56 \% \\
5.7 \% \\
108 \% \\
92 \%
\end{array}\right],\left[\begin{array}{c}
-29 \% \\
-59 \% \\
3.7 \% \\
152 \% \\
-41 \%
\end{array}\right]\right\}
$$


$x_{1}$ and $\Psi_{B}^{-1}\left(y \mid p_{B}^{\text {nom }}\right)$

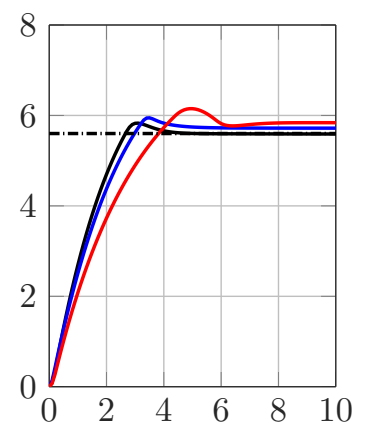

u

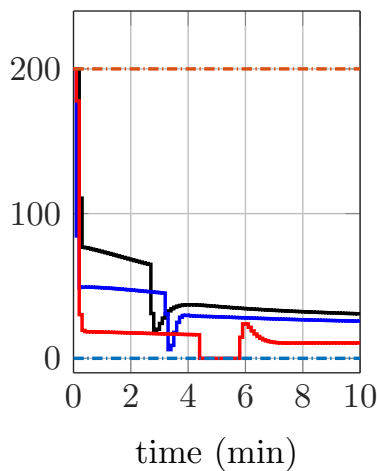

time $(\min )$
BIS
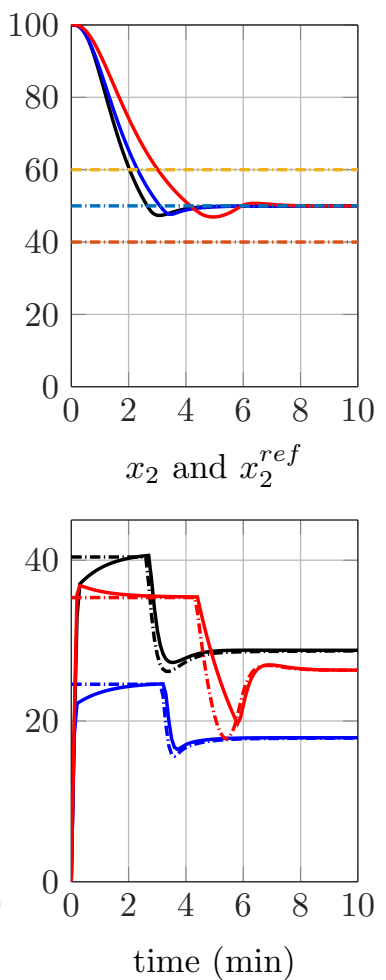

Fig. 1. Closed-loop simulations of three randomly sampled patients with the uncertainty levels given by (43). Notice the drastically different closed-loop Propofol injection profiles. Note also the integrator induced adaptation of the stationary values of $x_{1}$ in order to steer the BIS to its desired value despite of the uncertainties on the parameters of the BIS- $x_{1}$ static relation.

The closed-loop simulations for the three patients are given in Figure 1. One can easily notice the very different Propofol injection profiles for the three patients despite of the model-free design of the feedback law. The role of the integrator variable $e$ introduced in (36) can also be observed on the fact that the stationary values of $x_{1}$ are corrected and hence become patient-dependent so that the truly regulated BIS is steered to the desired value despite the unknown parameters of the specific patient.

\subsection{Probabilistic Certification}

In this section, the methodology described in Section 4.2 is applied. To do so, $n_{r}=100$ values of the response time have been taken, which are uniformly distributed on the interval $[1,6]$ min. The precision $\eta$ and the confidence parameter $\delta$ are both taken equal to $10^{-3}$. From Table 1 , it comes that the above choice leads to a number of samples $N=19299$ that is necessary to give the certification result. Figure 2 shows the histogram of the response time corresponding to the randomly sampled population of patients. From this figure, it comes clearly that a certifiable response time can be taken equal to $\bar{\tau}_{r}=4.9 \mathrm{~min}$. Let us state the result more clearly:
Histogram of $t_{r}$

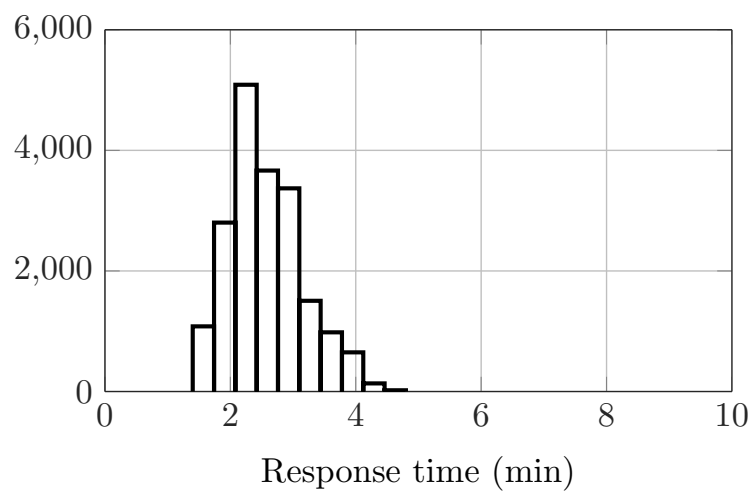

Fig. 2. Histogram of the response times for the 19299 sampled patients used in the computation of the probabilistically certified response time.

As far as the RMC described in Section 6.1 is relevant, under the proposed feedback, the probability that more than $0.1 \%$ of the patients BIS indicator remains outside the admissible domain after 4.9 min or gets their BIS lower than 40 is lower than 0.001 .

Note that the study can be conducted for different settings of the problem (different maximum injection intensity, different assumptions on the parameter dispersion, different models, different controller settings, etc.) The machinery including the structure of the feedback law remains identical.

\section{CONCLUSION AND FURTHER WORK}

In this paper, a complete framework for the design of certifiable dynamic output feedback law that can be used for Propofol-based anesthesia is proposed. The feedback formulation totally ignores the structure of the pharmacokinetic mechanisms. In spite of that, the certification results suggest that despite an extremely high variability of the patient response to the drug, a reasonable response time of less than 5 minutes can be probabilistically certified (with $99.9 \%$ of confidence) to be an upper bound of the time that would be necessary to steer any randomly sampled patient to the admissible 40-60 region of the BIS indicator.

The natural extension of the current work is to apply the proposed framework on real-patients under the supervision of an anesthetist in order to confirm the nice behavior of the control law in real-life situations.

\section{REFERENCES}

Alamir, M. (2015). On controlling unknown scalar systems with low gain feedback. Technical report, Technical report available on the open-collection hal-01215808.

Alamo, T., Tempo, R., and Camacho, E.F. (2009). Randomized strategies for probabilistic solutions of uncertain feasibility and optimization problems. IEEE Transactions on Automatic Control, 54(11), 2545-2559. 
Antsaklis, P. and Michel, A.N. (2007). A Linear Systems Primer. Birkhauser, Boston.

Bailey, J.M. and Haddad, W.M. (2005). Drug dosing control in clinical pharmacology. IEEE Control Systems Magazine, 25(2), 35-51.

Bickford, R.G. (1951). Automatic electroencephalographic control of anesthesia (servo-anesthesia). Electroencephalogr. Clin. Neurophys, 3, 83-86.

Charbati, S., Bracco, D., Mathieu, P.A., and Hemmerling, T.M. (2009). Comparison of four different display designs of a novel anaesthetic monitoring system, the integrated monitor of anaesthesia. British Journal of Anaesthesia, 103(5), 670-677.

Dumont, G. (2012). Closed-loop control of anesthesia a review. In 8th IFAC Symposium on Biological and Medical Systems, Budapest, Hungary, 373-378.

Gentilini, A., Rossoni-Gerosa, M., Frei, C., Wymann, R., Morari, M., Zbinden, A., and Schnider, T. (2001). Modeling and closed loop control of hypnosis by means of bispectral index (BIS) with isoflurane. IEEE Transactions on Biomedical Engineering, 48(8), 874889.

Ionescu, C.M., Keyser, R.D., Torrico, B.C., Smet, T.D., Struys, M.M., and Normey-Rico, J.E. (2008). Robust predictive control strategy applied for propofol dosing using BIS as a controlled variable during anesthesia. IEEE Transactions on Biomedical Engineering, 55(9), 2191-2170.

Mortier, E., Struys, M., Smet, T.D., Versichelen, L., and Rolly, G. (1998). Closed-loop controlled administration of propofol using bispectral analysis. Anaesthesia, 53(8), $749-754$

O'Hara, D., Bogen, D., and Noordegraaf, A. (1992). The use of computers for controlling the delivery of anesthesia. Anesthesiology, 77, 563-581.

Ritchie, R.G., Ernst, E.A., Pate, B.L., Pearson, J.D., and Shepherd, L.C. (1987). Closed-loop control of an anesthesia delivery system: Development and animal testing. IEEE Trans. Biomed. Eng., 34(6), 437-443.

Ross, J., Wloch, R., White, D., and Hawes, D. (1982). Servo-controlled closed-circuit anaesthesia. A method for the automatic control anaesthesia produced by a volatile agent in oxygen. IEEE Transactions on Automatic Control, 55(11), 229230.

Schwilden, J., Schuttler, H., and Stoeckel, H. (1987). Feedback control of methohexital anesthesia by quantitative eeg analysis in humans. Anesthesiology, 67(3), 341-347.

Smet, T.D., Struys, M.M.R.F., Greenwald, S., Mortier, E.P., and Shafer, S.L. (2007). Estimation of optimal modeling weights for a Bayesian-based closed-loop system for propofol administration using the bispectral index as a controlled variable: A simulation study. Anesth. Analg., 105, 1629-1638.

Vishnoi, R. and Roy, R.J. (1991). Adaptive control of closed-circuit anesthesia. IEEE Transactions on Biomedical Engineering, 38(1), 3947.

Zabi, S., Queinnec, I., Tarbouriech, S., Garcia, G., and Mazerolles, M. (2015). New approach for the control of anesthesia based on dynamic decoupling. In Proceedings of the 9th IFAC Symposium on Biological and Medical Systems, Berlin, Germany, pp. 511-516. 